\title{
Study of electrooculography signal acquisition sites for assistive device applications
}

\author{
Karthik Raj V* \\ Assistant Professor, Department of Biomedical Engineering, Vel Tech Rangarajan Dr. Sagunthala R \& D Institute of \\ Science and Technology, No.42, Avadi-Vel Tech Road, Vel Nagar, Avadi, Chennai - 600062, Tamilnadu, India
}

Received: 13-June-2021; Revised: 24-August-2021; Accepted: 26-August-2021

(C)2021 Karthik Raj V. This is an open access article distributed under the Creative Commons Attribution (CC BY) License, which permits unrestricted use, distribution, and reproduction in any medium, provided the original work is properly cited.

\begin{abstract}
Electrooculography (EOG) is a technique that involves the measurement of the corneo-retinal standing potential of the eye. The human eye acts as a dipole between the cornea (positive potential) and the retina (negative potential), creating an electric field around the eyeball. The resulting electric signal obtained from this field is called electrooculogram. These signals, generated by eye movements, could be measured by employing different electrode placement configurations for the acquisition. The properties of these signals change depending on the number and placement of the electrodes. The study conducted here describes the EOG signal acquisition using new electrode placement configurations that employ fewer facial electrodes placed on the patient. Three pre-gelled disposable electrodes were utilized for this purpose. Only one electrode was placed in a facial location, enhancing patient comfort during the acquisition procedure. To support this study, a lowcost signal acquisition hardware was developed. Using active filtering and amplification, appropriate signal processing techniques were executed upon the horizontal EOG signal acquired to reduce noise and interference due to external conditions. Hence, this paper presents the findings of new electrode placement sites for the acquisition of EOG signals which could be used for assistive device applications while restricting the number of facial electrodes to one. Most of the studies regarding the EOG signal acquisition had been using all electrodes in the face region. In contrast, we reduced the number of electrodes in the facial region, thereby providing patient comfort. The comparison was made mainly on the acquired data from these new locations to discover the configuration with the optimal signal response using data obtained from seven healthy subjects. The amplitude values were being compared from the new locations with the standard acquisition sites. The findings of this study were found to have a productive result. The total gain of the system required for new electrode placement configurations was two times more than the total gain required for standard acquisition sites, and also, the amplitude was less but can be helpful for assistive device applications. The average peak to peak amplitude value of the EOG signal for the new site was approximate 1.25 volts.
\end{abstract}

\section{Keywords}

Horizontal EOG, Facial electrodes, Pre-gelled disposable electrode, Signal processing.

\section{Introduction}

Biomedical signals like electroencephalogram, electromyogram and electrooculogram are used in assistive device applications for patients suffering from speech disorders, spinal cord injury and hand amputations. All these signals are low frequency and low amplitude signals [1]. When we try to pick these signals from our body, many artifacts or noises are present in the signal, which makes the signal-to-noise ratio poor. Among these signals, the electrooculogram is the most straightforward bioelectrical signal with a high signal-to-noise ratio and can be used to control several applications $[2,3]$.

\footnotetext{
*Author for correspondence
}

To pick the biomedical signal from our body, the placement of electrodes in the body is a vital factor [1].

The estimation of eyeball orientation can be done based on the varying voltage amplitude, measured across the electrode through the Electrooculography (EOG) technique. Electric fields are generated around the eyeball due to the eyeball's dipole nature between the cornea (positive potential) and the retina (negative potential) [1]. Generated EOG signals are picked up by the electrodes placed on the facial locations around or near the eye. Depending on the position of the electrode with respect to the eyeball movement, the voltage generated is positive or negative and zero when looking straight at an angle of $90^{\circ}$. Typically in humans, the EOG signal ranges from 0.05 to 3.5 milli- 
volt $(\mathrm{mV})$ with a frequency range of 0.1 to 20 hertz (Hz) [1].

Several electrode configurations are employed for the acquisition of the EOG signal. Depending on the number of electrodes and their placement, the properties of the signal change accordingly. In a recent study, peculiarities of different electrode configurations have been analysed using a low-cost hardware device specifically for medical applications [4]. The EOG measurement recordings are further used for diagnostic purposes and investigation of the proper functioning of the ocular motor system. With the advancement in research and design methodology, its potential application is in the field of human assistive technology in the case of paralysis. Considering a quadriplegic patient where the patient is paralyzed from the upper and lower limb, the EOG signal can be used as a mode of communication to interact with their caregivers and a monitoring system for the patients [5]. Cost-effective hardware has been developed for patients with paralysis, which facilitates them to control the home appliances and activ ate them for daily activities [6]. The placement of electrodes for horizontal and vertical EOG signal acquisition usually involves seven electrodes in the face region [7]. If we are picking the horizontal EOG signal alone (left and right movement), we require a minimum of three electrodes in the face region to pick the signal [5]. Usually, everyone uses all the electrodes in the face region. In contrast, in this study, only one electrode is used in the face region to provide patient comfort for picking the EOG signal for the human assistive technology.

\section{Literature review}

Human-machine interfacing based on EOG has been utilized for designing a smart wheelchair electric system for disabled people that enables themto control and guide their movement [8-10]. Many advanced signal processing techniques have been incorporated for the past few decades to obtain an accurate and precise signal. Many factors contribute to the variation in the electrooculogram reading, such as electrode placement, head movement, blinking, lightening conditions, etc. [4, 8]. EOG based systems have been designed for controlling the virtual keyboard and mouse curs or [11-13]. Recently, some researchers have used a stable EOG interfacing unit for controlling electric wheelchairs for disabled people [14-18]. Signal de-noising methods such as wavelet transform technique, amplification procedure and artifacts reduction techniques have been employed to get an excellent noise-free EOG signal [5, 19]. Vertical EOG and horizontal EOG signals have been picked from the subject for various purposes [20-23]. By picking the vertical EOG signal, a low-cost driver alertness system has been designed for preventing road accidents by quickly alerting the driver if he feels drowsiness to ensure their safety [24]. By acquiring the vertical and horizontal EOG, patients with severe motor disabilities have communicated with, their surrounding people using EOG based speller [25]. Eight directional eye movement classification algorithm has been an effective way to analyse the aftermath effect of noise in EOG signals [26-28]. Several human-machine interface technologies utilizes horizontal EOG signal, which has been used as a control strategy for a mobility aid [29]. Feature extraction techniques have been performed for analysis of the signal with minimum redundancy and maximum relevant features to improve the communication based on the EOG signal for disabled people [30-33]. Generally, for EOG signal acquisition techniques, a three-electrode system has been employed for communication and control purposes of assistive devices [34]. However, with the increase in the number of electrodes, the discomfort caused to the patient also increases. Over the recent years, EOG based devices such as human-computer interface, robotic assistive technology, rehabilitation aid, etc., have been gaining popularity due to their potential application [35-39]. With the growing demand, developing a low-cost EOG signal acquisition device would serve the need and prioritize patient comfort while ensuring efficiency in the data acquisition.

This paper gives a detailed insight into the new electrode placement sites for EOG signal acquisition that would enhance patient comfort by reducing the number of facial electrodes to one which could be used for assistive device applications. Four new locations for electrode placement are tested for this purpose. In our study, the horizontal EOG signal is recorded, as it provides less interference than the vertical EOG signal. A low-cost signal acquisition hardware is developed and proper signal conditioning is incorporated to reduce noise and interference due to external conditions. Acquired data are then interfaced with a computer for logger recording using the biomedical workbench of Laboratory Virtual Instrument Engineering Workbench (LabVIEW) software. With the horizontal movement of the eyeball, the EOG signal is generated. The strength of the acquired signal that is obtained from these new sites is analysed to find the best locations with the optimum response. In short, the main objectives of this study are as follows - 
- To find new electrode placement sites for EOG signal acquisition

- To minimize the number of electrodes used in the facial location of the patient while restricting it to one electrode on the face

- To perform a comparative study among the acquired signal data from the new electrode placement locations in terms of the output yield at each site of the new location

\section{Methods}

The EOG system was formed by developing a circuit implementing active filters and amplifier circuits, thus allowing the acquisition of an effective EOG signal that could be viewed using a Digital Storage Oscilloscope (DSO), or the National Instruments -
Educational Laboratory Virtual Instrumentation Suite (NI-ELVIS) kit and LabVIEW software.

\subsection{Block diagram}

Figure 1 shows the block diagram of the EOG system. The EOG signal was given to a voltage follower for impedance matching. Since the EOG signal amplitude was very low, it was given to an instrumentation amplifier for amplifying its voltage after the voltage follower circuit [5, 25]. Also, the instrumentation amplifier circuit was having a high Common-Mode Rejection Ratio (CMRR). After the instrumentation amplifier, the signal was given to the high-pass filter and low-pass filter circuits for getting the horizontal EOG signal alone while rejecting the other frequency signals. After this, the signal was passed to a variable non-inverting amplifier to get the appropriate signal voltage to be visualized in the oscilloscope.

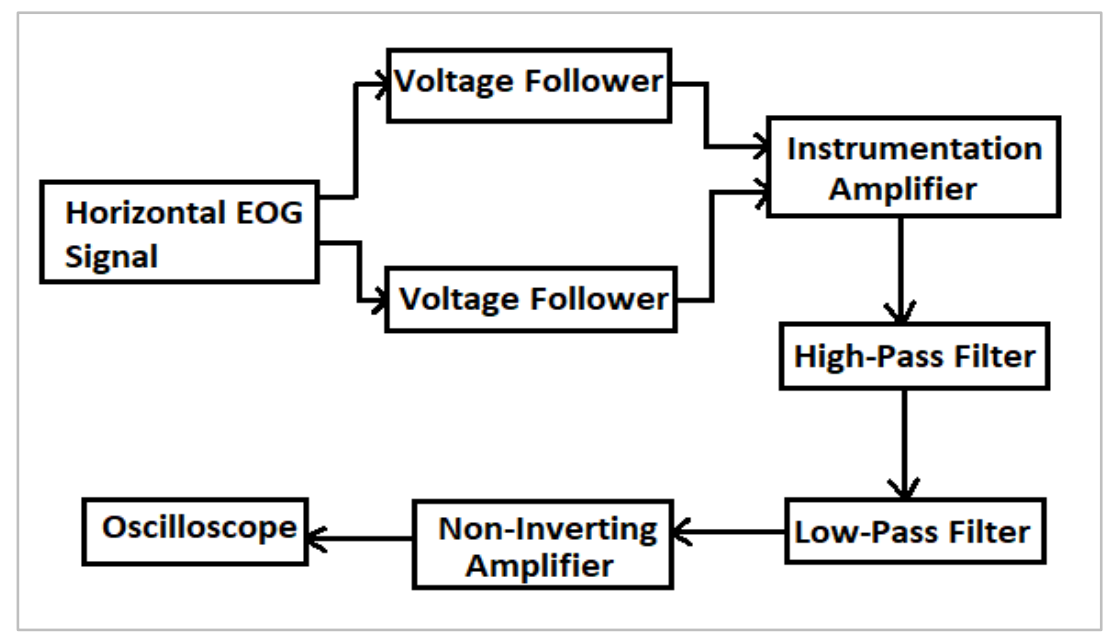

Figure 1 Block diagram of the EOG system

Figure 2 shows the detailed circuit diagram. The gains and cut-off values were discussed further along with each sub-circuit. An Operational Amplifier (op-amp) based amplifier circuit with a voltage gain value of 1 represents a voltage follower circuit. Inputs from electrode-1 (-) and electrode-2 (+) are fed to their respective follower circuits depicted by the boxes 'A' and 'B' in Figure 2, following which they supply to the instrumentation amplifier depicted by ' $C$ ' at the subsequent stage. Voltage follower circuits were used to prevent impedance mismatch at the inputs.

The pre-amplifier implemented using an instrumentation amplifier performs the operation of strengthening an acquired signal by amplifying it with a user-set gain and eliminating the common noises from the differential input is shown in Figure 2 as ' $\mathrm{C}$ '. Here, an AD620 instrumentation amplifier was used 991 with a gain $(G)$ value of 250 . Thus, a value of $R_{1}$ was obtained as $198.39 \Omega$ approximated to $200 \Omega$ by using Equation 1.

$\mathrm{R}_{1}=(49400) /(\mathrm{G}-1)$

Movement of the subject produced motion artifacts that cause baseline wandering. A second-order Butterworth high-pass filter was implemented to eliminate this effect and block low-frequency noise. In the circuit diagram, Figure 2, it is shown as 'D'. A high-pass filter is an active filter that responds only to signals above a set cut-off frequency. The filter designed in this circuit had a cut-off frequency (fc) of $2 \mathrm{~Hz}$. A Butterworth filter has been chosen for its ideal response characteristics in biomedical applications [5]. For the required frequency, capacitance $C_{1}$ and $C_{2}$ were assumed as $10 \mu \mathrm{F}$ (microfarad) and corresponding resistors $R_{2}$ and $R_{3}$ were obtained as 
$7.95 \mathrm{k} \Omega$ (kiloohm) rounded off as $8 \mathrm{k} \Omega$ by using Equation 2. The gain of the high-pass filter was calculated as 1.586 by using the standard Equation 3 for the Butterworth filter. In Equation 2, $\mathrm{R}=\mathrm{R}_{2}=\mathrm{R}_{3}$ and $\mathrm{C}=\mathrm{C}_{1}=\mathrm{C}_{2}$, which are shown in Figure 2 marked as ' $\mathrm{D}$ '.

$\mathrm{fc}=1 /(2 \pi \mathrm{RC})$

$\mathrm{DF}=2-\mathrm{R}_{5} / \mathrm{R}_{4}$

In Equation 3, DF stands for the damping factor. The damping factor value of a Butterworth filter was considered as 1.414. After substituting the value of the damping factor in Equation 3. It could be modified as Equation 4.

$\mathrm{R}_{5} / \mathrm{R}_{4}=0.586$

By using the gain formula for a non-inverting amplifier and the value obtained in Equation 4, the gain of the high-pass filter was obtained as 1.586 .

A low-pass filter is a type of filter that allows only frequencies lower than the set cut-off frequency in its passband. In this system, two second-order low-pass Butterworth filters were cascaded to form a fourthorder low-pass filter. This part is shown in Figure 2 as 'E'. The frequency of horizontal channel EOG signals ranges from 2 to $16 \mathrm{~Hz}$. Therefore, we had set the cutoff frequency (fc) to $10 \mathrm{~Hz}$. The output of the first low pass-filter was fed to the input of the second filter. For $10 \mathrm{~Hz}$ cut-off, $\mathrm{C}_{3}$ and $\mathrm{C}_{4}$ were assumed as $0.1 \mu \mathrm{F}$. Resistors $R_{6}$ and $R_{7}$ were calculated as $159.154 \mathrm{k} \Omega$ by using Equation 2. The resistor values were rounded off as $160 \mathrm{k} \Omega$. Here $\mathrm{R}=\mathrm{R}_{6}=\mathrm{R}_{7}=\mathrm{R}_{11}=\mathrm{R}_{12}$ and $\mathrm{C}=\mathrm{C}_{3}=$ $\mathrm{C}_{4}=\mathrm{C}_{5}=\mathrm{C}_{6}$ which are shown in Figure 2 marked as ' $E$ '. The gain of each $2^{\text {nd }}$ order low-pass filter was considered as 1.586 since it was a Butterworth filter. The gain of the cascaded $4^{\text {th }}$ order filter was given by $1.586 \times 1.586$, which was 2.515 .

At this point, the overall gain of the system was 997.197. This was achieved by multiplying the gain values of the filters and multiplying the resultant with the instrumentation amplifier gain. The non-inverting amplifier represents the designed system's final stage, which is represented by part ' $\mathrm{F}$ ' in Figure 2. The task of amplifying the received filtered signal to a sufficient level was done by the non-inverting amplifier using a variable gain set-up. The output of the $4^{\text {th }}$ order low pass filter was fed as the input to this amplifier. The gain of the non-inverting amplifier $\left(\mathrm{G}_{\mathrm{n}}\right)$ was taken as 11 and 23, respectively. Fixing the input resistance $R_{17}$ as $1 \mathrm{k} \Omega$, we obtained the values of feedback resistance $R_{16}$ as $10 \mathrm{k} \Omega$ for a gain value of 11 and $22 \mathrm{k} \Omega$ for a gain value of 23 by using Equation 5 .

$\mathrm{G}_{\mathrm{n}}=1+\left(\mathrm{R}_{16} / \mathrm{R}_{17}\right)$

Power supply for the circuit was given by using two 9volt (V) batteries or through the variable power supply option of the NI-ELVIS kit to achieve a dual supply voltage of \pm 9 V. Figure 3 shows the experimental setup used for acquiring signals from the patients.

In Figure 3, each major component is marked as 'A', 'B', 'C', 'D', 'E', 'F', and 'G'. They collectively represent the front panel of the entire sys tememployed to acquire the signal. Component ' $\mathrm{A}$ ' is the computer system paired with NI-ELVIS marked by the letter ' $E$ '. The computer system constitutes the main signal recording system. NI-ELVIS is used to supply voltage $( \pm 9 \mathrm{~V})$ to the EOG acquisition circuit marked by the letter ' $\mathrm{D}$ '. The DSO is represented by the letter ' $C$ ' and offers the mode of visualizing waveforms. Component ' $F$ ' (not included in the actual system as it is used only for testing) is an arrhythmia simulator used only to test the circuit before its actual usage. The component represented by the letter ' $G$ ' is the probe cable wire that attaches to the electrodes on the patient to supply the potentials to the voltage followers in the circuit given by the letter ' $D$ '. Also, the letter 'B' placed in the monitor of the computer is the Biosignal logger and the player used for real-time logging signals for a given amount of time and is a part of the biomedical workbench of the LabVIEW software. 


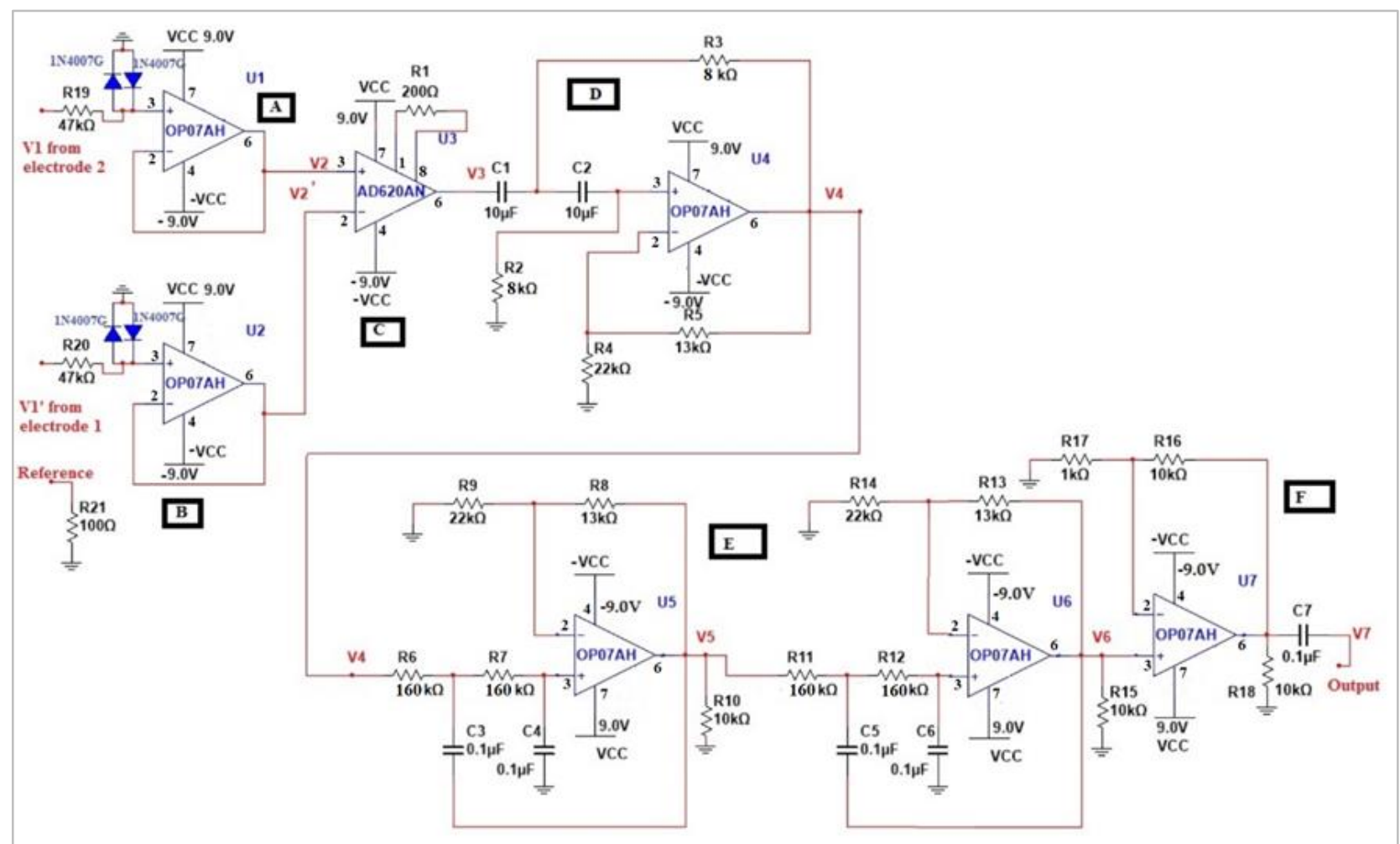

Figure 2 Detailed circuit diagram

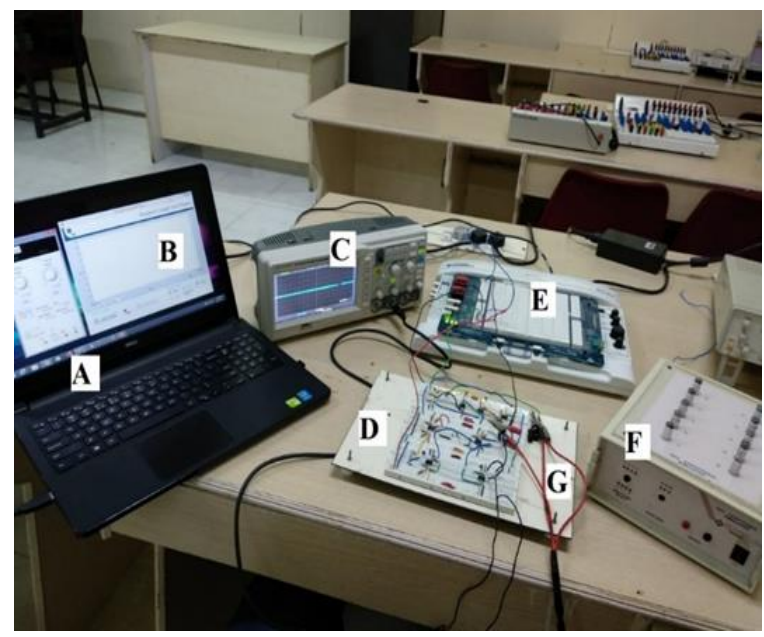

Figure 3 Experimental setup for signal acquisition

\subsection{Electrode placement}

The EOG system used three electrodes to acquire the signal of interest. Pre-gelled disposable surface electrodes were used to pick up the signals from the appropriate positions. According to the electrode system taken as shown in Figure 4, electrode 2 plays the role of positive (+) electrode, whereas electrode 1 plays the role of negative (-) and the reference electrode contributes the ground potential.
3.2.1Basic electrode positions

These positions involve the primary positioning of positive (+) and negative (-) electrodes at the right and left temples, respectively, while varying the reference electrode position alone. Different locations of the reference electrode placement give rise to 9 different positions, sequentially named from P1, P2, P3, P4, P5, P6, P7, P8 and P9. Figure 4 shows positions P1, P2, $\mathrm{P} 3, \mathrm{P} 4, \mathrm{P} 5$ and P6 where the reference electrode is placed on the forehead between the eyebrows, on the left earlobe, on the right earlobe, behind the left earlobe, behind the right earlobe, and the left side of the thyroid respectively. Figure 5 shows the positions P7, P8 and P9 where the reference electrode is placed on the right side of the thyroid, on the left and the right wrists, respectively.

3.2.2 New electrode positions

Through careful experimentation, the following positions were obtained where the locations of the positive (+) and negative (-) electrodes are no longer fixed. The reference electrode was also moved to different locations, giving four possible combinations of electrode placement named N1, N2, N3 and N4, respectively. These configurations were independent of the basic positions used to obtain general EOG signals. By placing the positive (+) electrode at the right temple and the negative (-) electrode at the base 
of the jaw below the left ear while simultaneously placing the reference at the right earlobe, the new position N1 is obtained, which is shown in Figure 6. Then, keeping the positive (+) electrode at the right temple and changing the position of the negative (-) electrode to behind the left earlobe and the reference to behind the right earlobe, position N2 is achieved, which is shown in Figure 6. Switching the side of the positive (+) electrode to the left, two positions are accomplished. N3 is obtained by placing the positive (+) electrode at the left temple, the negative (-) electrode behind the left ear and the reference electrode behind the right ear as shown in Figure 6. Keeping the positive (+) electrode at the left temple and interchanging the positions of the negative (-) electrode and reference electrode such that the negative (-) electrode is behind the right ear and the reference electrode is behind the left ear, position N4 is obtained as shown in Figure 6. Figure 7 shows an example of the electrode placement on a live subject. It can be noted from Figure 6, that in any of the four configurations, only one electrode is placed directly on the face. This is done to enhance patient comfort.

\subsection{NI-ELVIS}

The NI-ELVIS is an engineering laboratory solution designed for academic purposes. It is a modular workstation that includes several virtual lab instruments such as an oscilloscope, a multimeter, a function generator, a variable power supply and more. The circuit implemented for this work was powered by the ELVIS kit by pairing it with a computer via the universal serial bus. Pins 48, 49, and 50 of the variable power supply boards were used to supply voltage to the circuit. To log the signals acquired, the analog input channels of the kit were paired with the circuit. The kit is displayed as component E in Figure 3.

\subsection{Biomedical workbench}

The biomedical workbench is an extended toolkit for LabVIEW software specially designed for biomedical applications such as image analysis and signal acquisition. The application is directly correlated with any national instruments data acquisition hardware device, for e.g., National Instruments - Data Acquisition (NI-DAQ) and NI-ELVIS. It makes it possible to visualize real-time Biosignals acquired through the sensors coupled in the circuit. The Biosignal logger and player were used for logging EOG signals for one minute per subject. The circuit was implemented and the resultant waveforms were saved using a DSO and data recordings were performed using the Biosignal logger and player in the biomedical workbench.

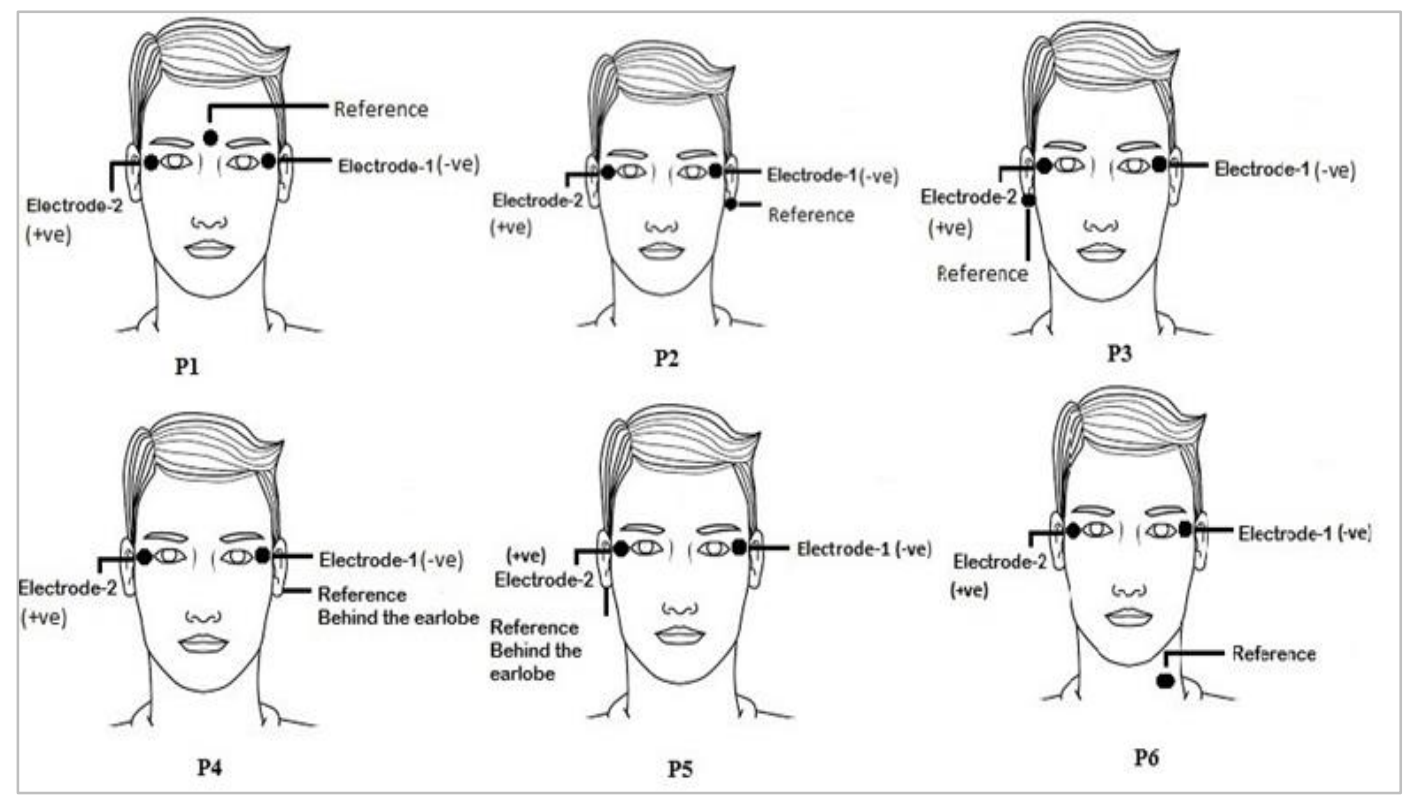

Figure 4 Description of positions P1, P2, P3, P4, P5 and P6 


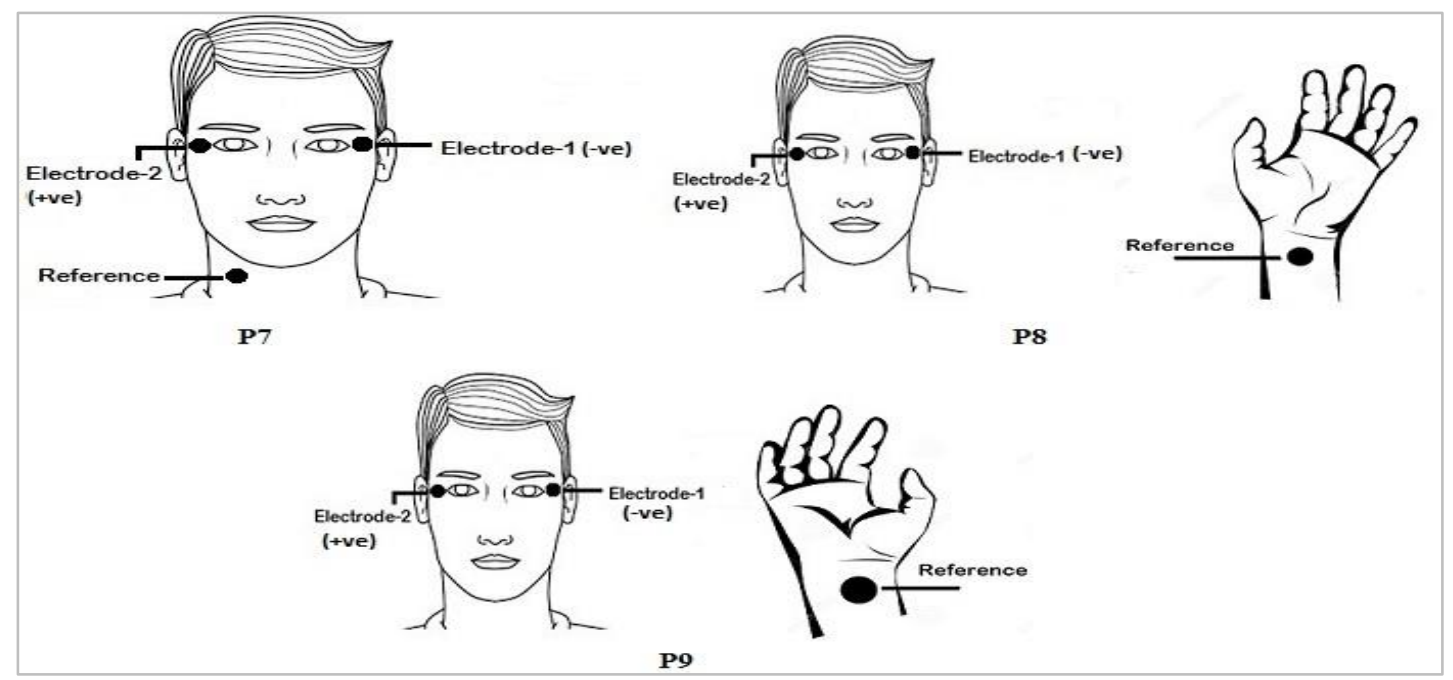

Figure 5 Descriptions of positions P7, P8 and P9

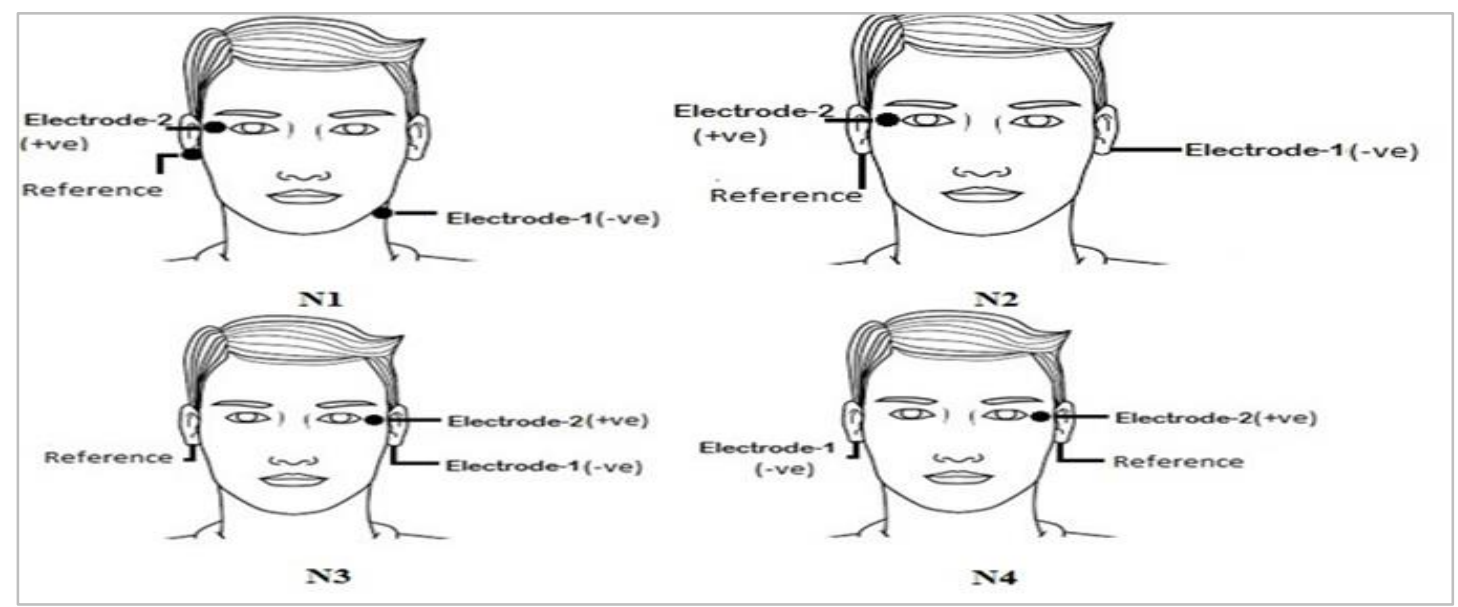

Figure 6 Description of positions N1, N2, N3 and N4

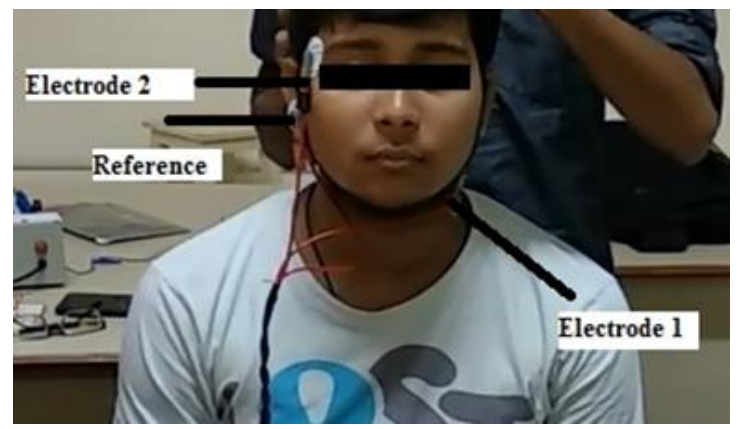

Figure 7 Electrode placement on a live subject

\section{Results}

This section specifies the various signal outputs obtained from the designed EOG acquisition circuit for different positions as shown in Figure 8. The system was successfully tested on seven healthy subjects from whom the signal was acquired while in a sitting position with arms resting on the lap. Data from each of the subjects were included in the study and is illustrated as a series of signal peaks for both left and right movements at each different electrode placement configuration in Figure 8. Figure 8 shows the left and right eye movement for the various configurations P1, P2, P3, P4, P5, P6, P7, P8, P9, N1, $\mathrm{N} 2, \mathrm{~N} 3$ and N4, which are acquired from healthy subjects.

The basic electrode positions for Figure 4 and Figure 5 are derived by keeping constant the positive (+) and negative (-) electrodes while changing the placement of only the reference electrode. The new electrode placement configurations involved the placement of only one electrode on the face at a time. These positions are named N1, N2, N3 and N4, as shown in Figure 6. 
The column charts illustrate a visual representation of the numerical data acquired from each of the seven subjects. This data was obtained by calculating the peak-to-peak amplitudes of the waveforms manually using the screen grid and comparing it to the scale setting. The number of boxes on the vertical axis of the DSO that was covered by the signal from the bottom to the top peak point was multiplied by the value of each single box gave the resultant values in volts. Four values are depicted here - left movement, the return movement to the center, right movement and the corresponding return movement to the central position. Each of these values is represented by a different color. Figures 9, 10, 11, 12, 13, 14, 15, 16,
$17,18,19,20$ and 21 show the peak-to-peak voltage data obtained for all seven subjects for all electrode positions. Each of these values is in volts and represents the peak-to-peak amplitude of the EOG signal. These values clearly describe that the EOGhad been successfully acquired from the subject through pre-gelled disposable electrodes. The system had successfully picked up and performed signal conditioning on the signals. Data acquired from positions P1 to P9 were taken as a final stage amplifier gain value of 11, whereas data acquired frompositions $\mathrm{N} 1$ to $\mathrm{N} 4$ were taken with a gain value of 23 at the final stage amplifier.

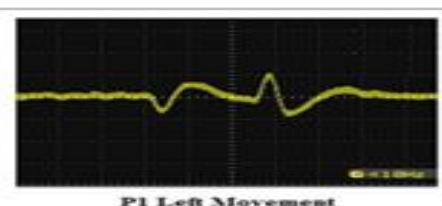

P1 Keff Movernent

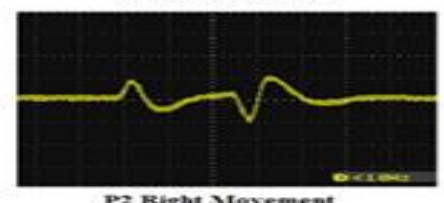

P2 Riblit Mavement

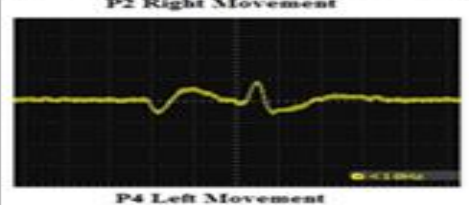

PS Leet Mtoverment
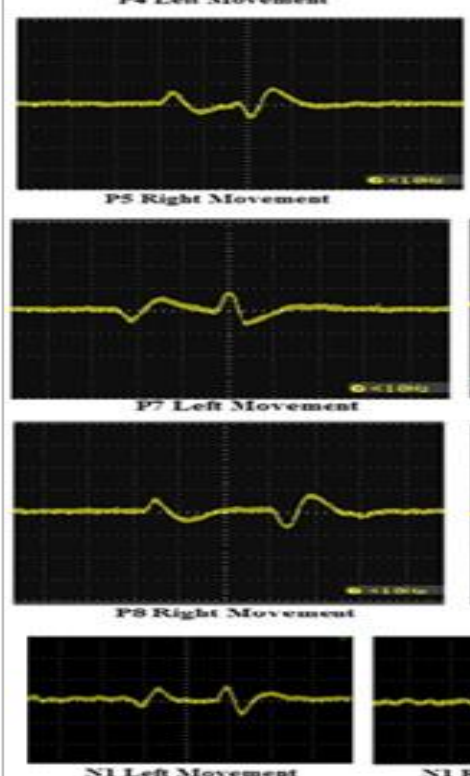

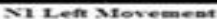
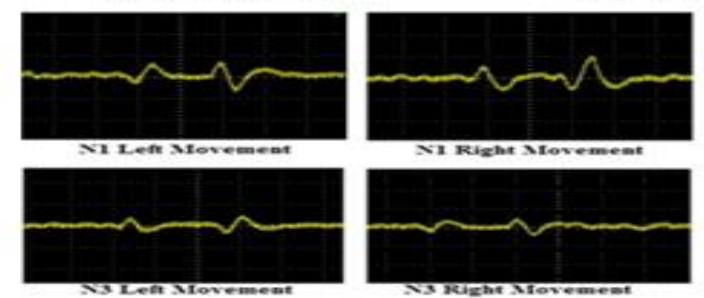

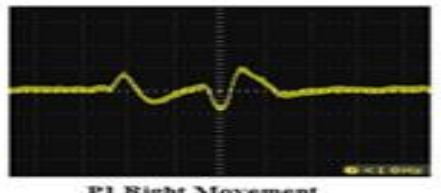

Pl Right Sxovement

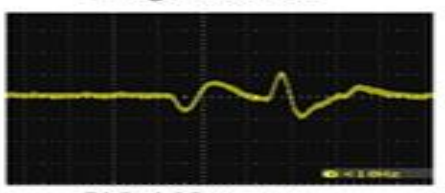

Pa Lefr Mxovemeat
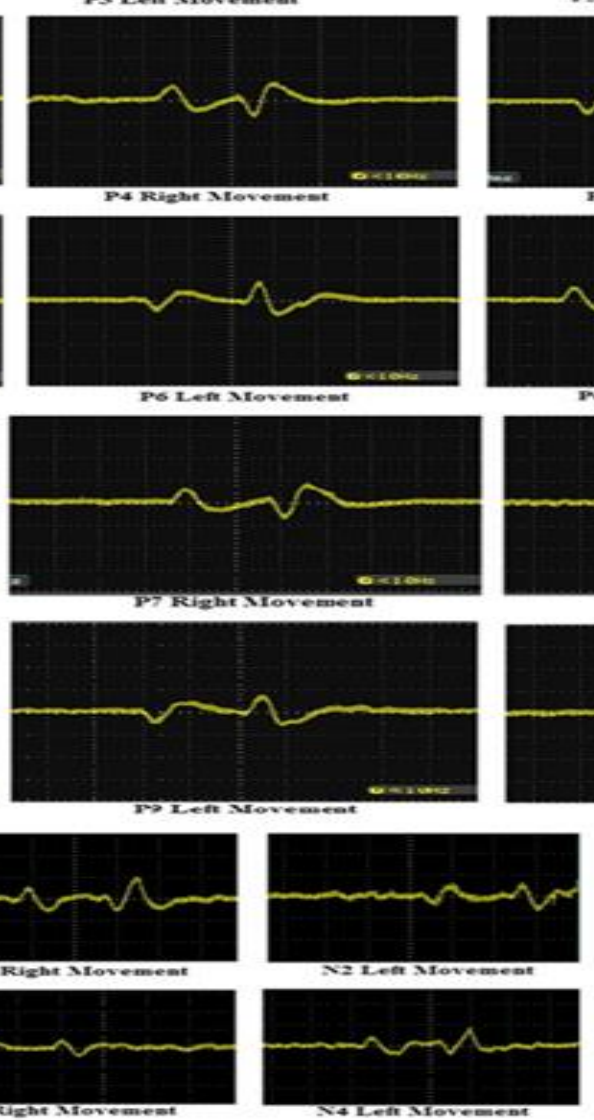

sutert sroweent

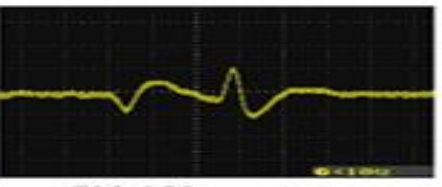

Pz Lefr Mrovement.
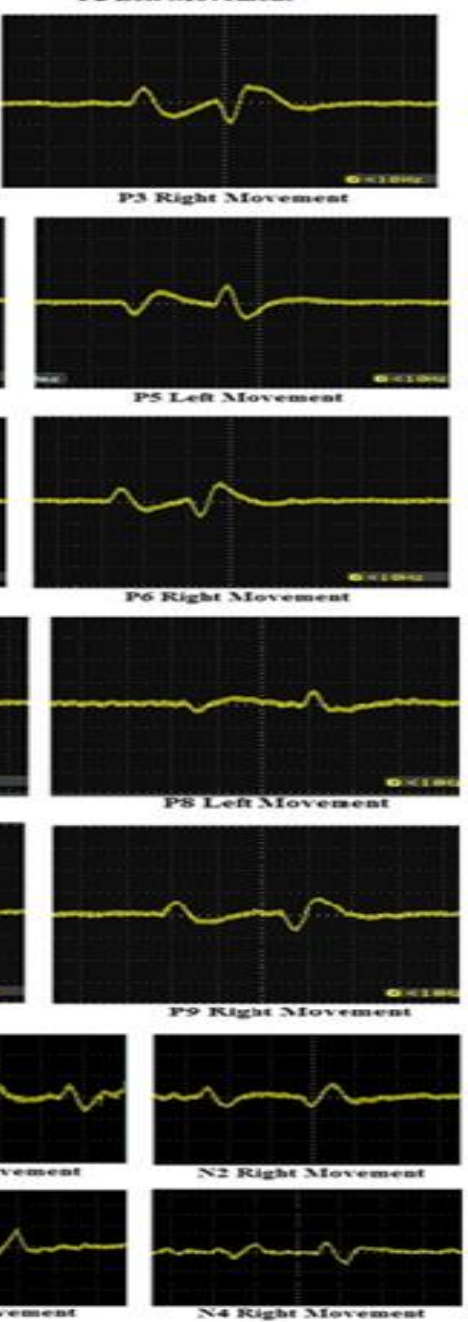

Figure 8 EOG signals for various positions 


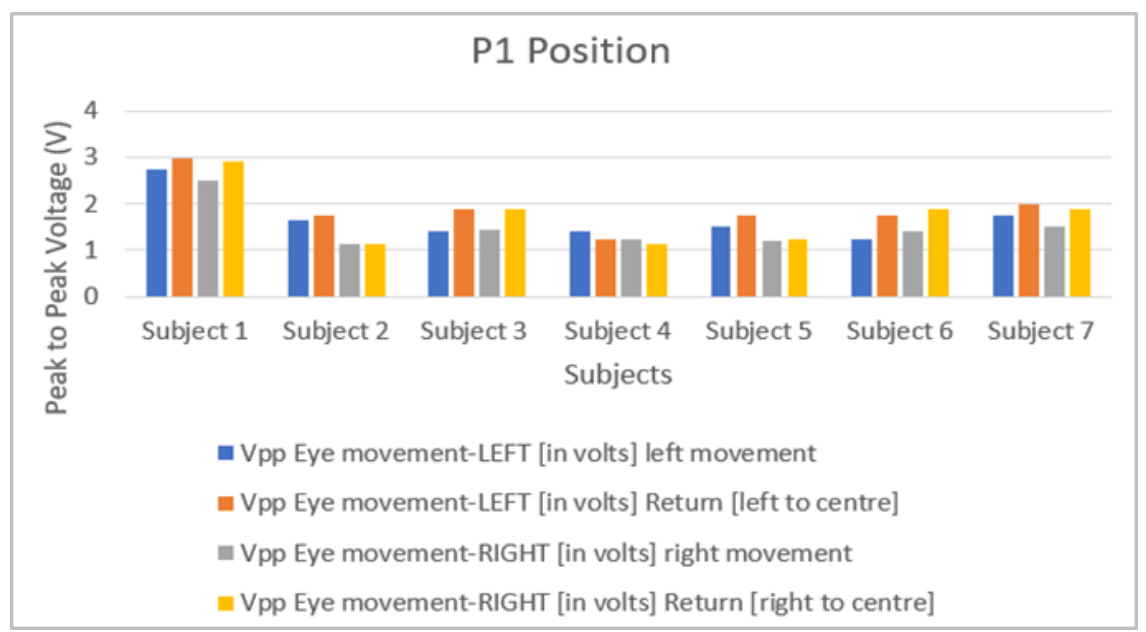

Figure 9 Data for P1 position

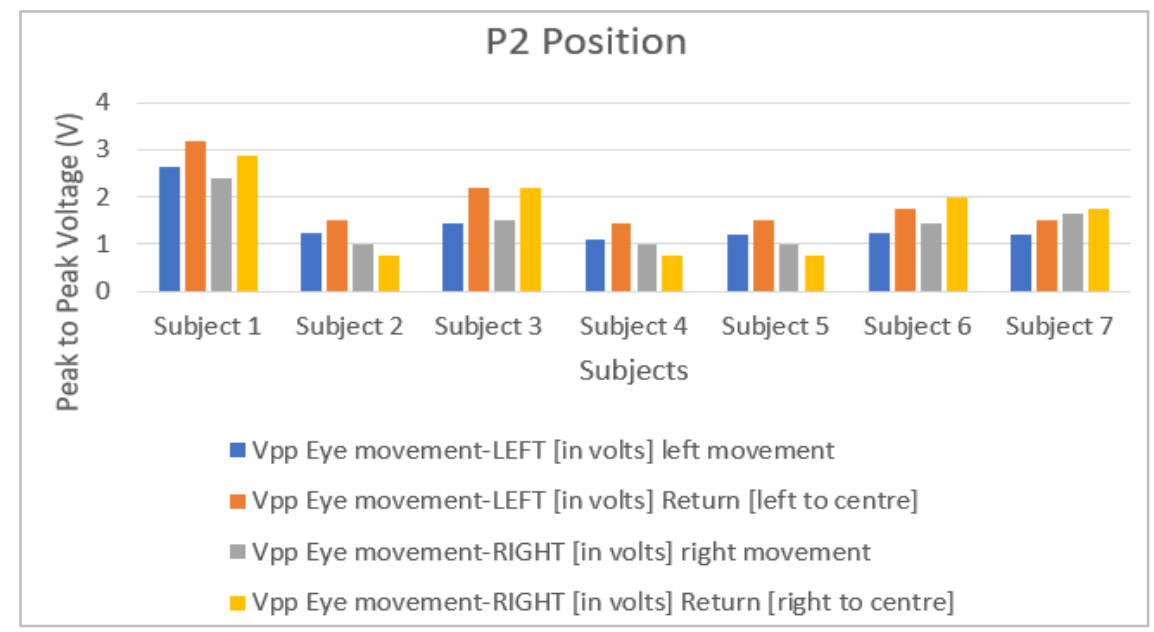

Figure 10 Data for P2 position

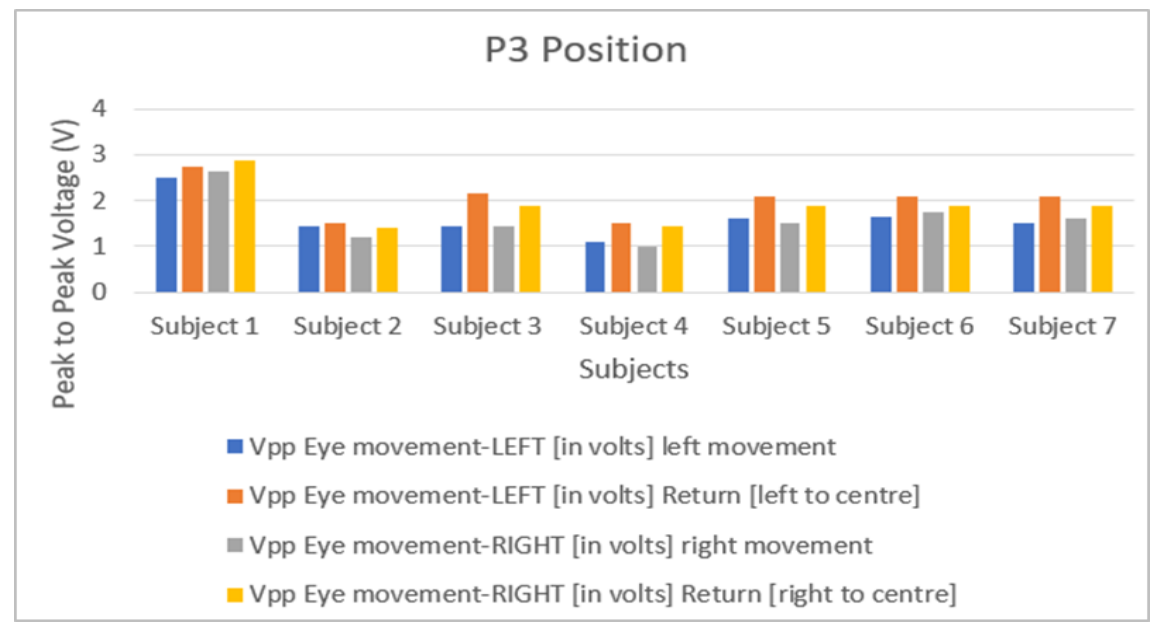

Figure 11 Data for P3 position 
Karthik Raj V

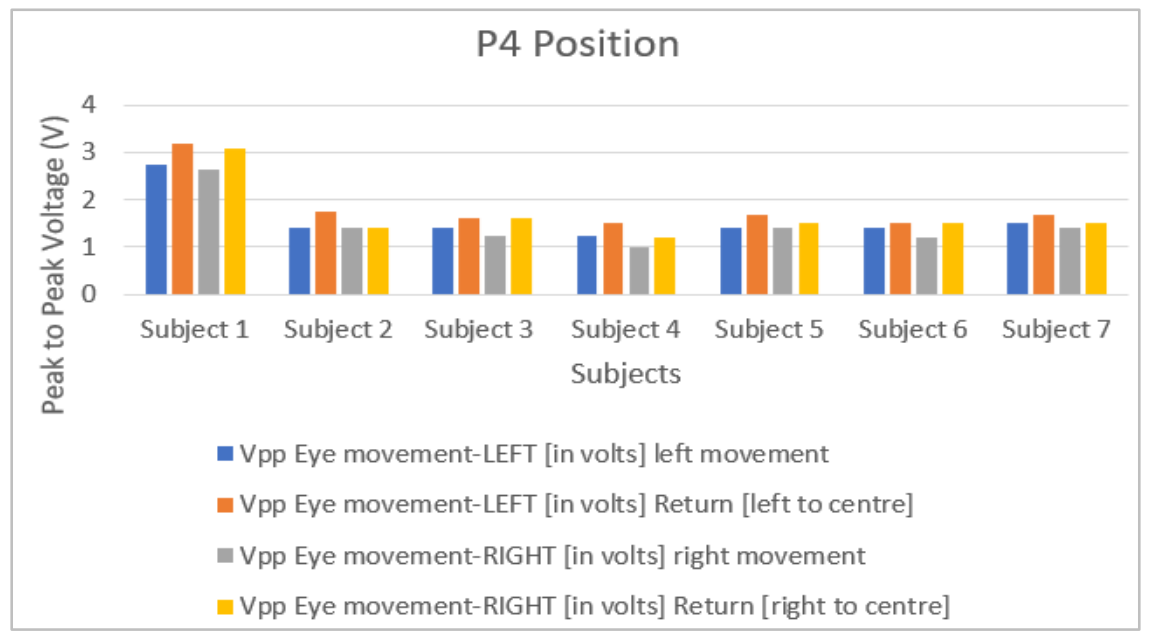

Figure 12 Data for P4 position

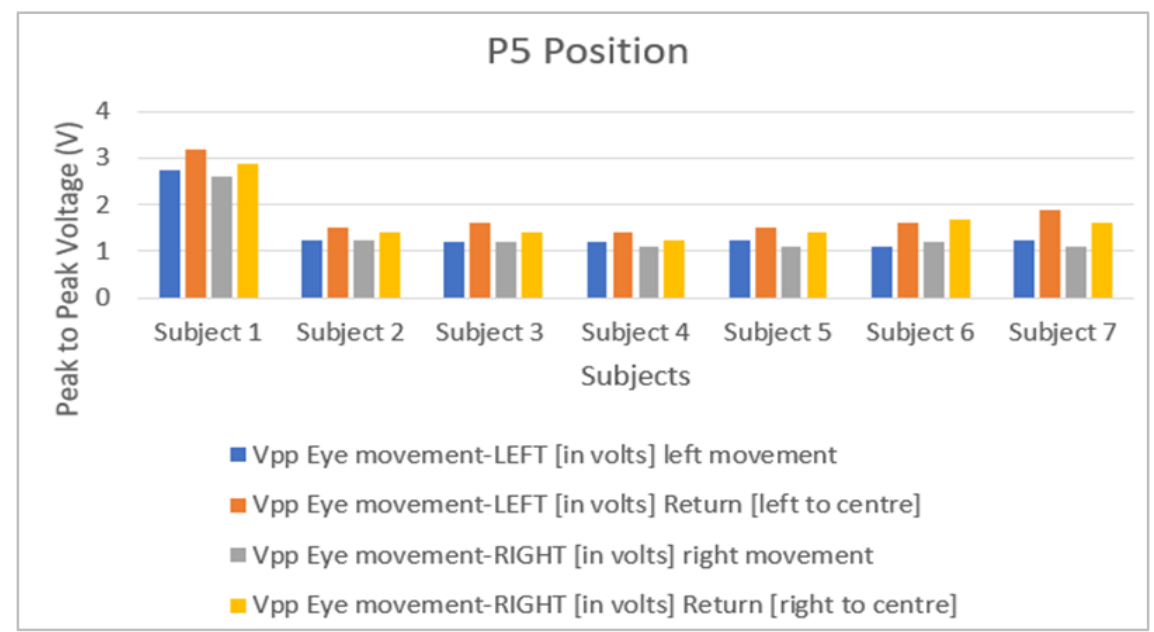

Figure 13 Data for P5 position

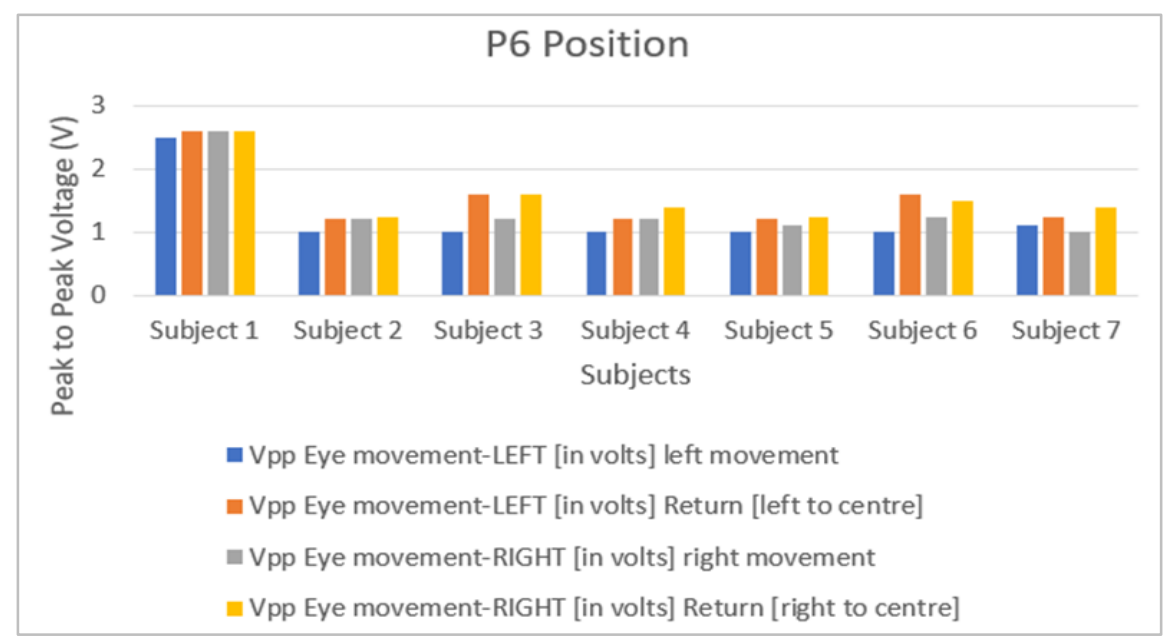

Figure 14 Data for P6 position 


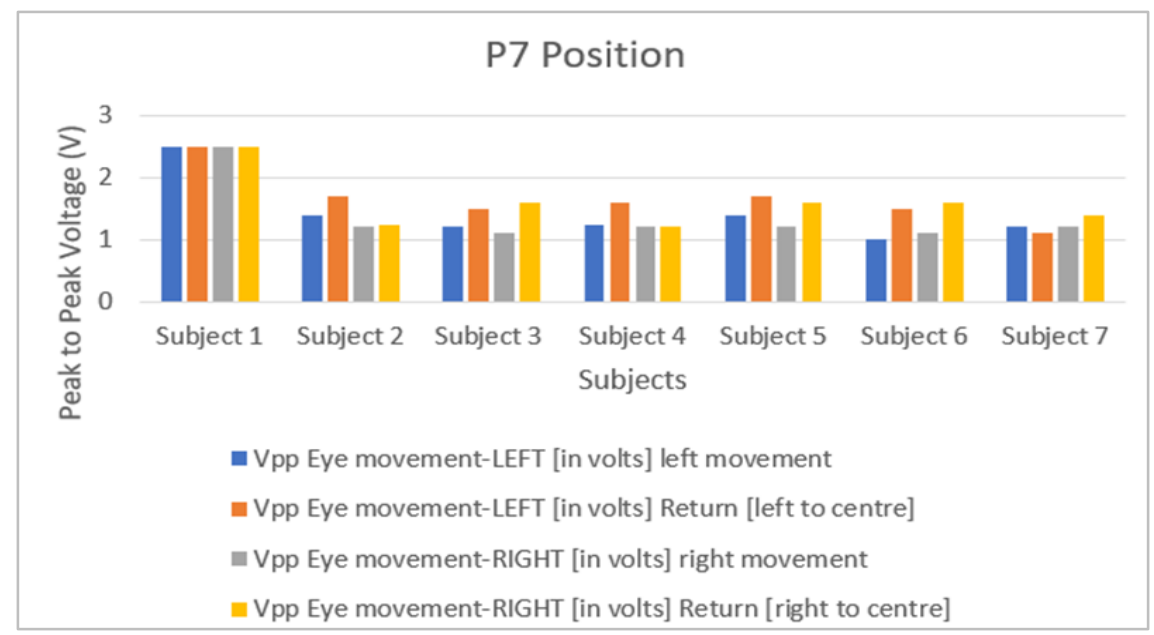

Figure 15 Data for P7 position

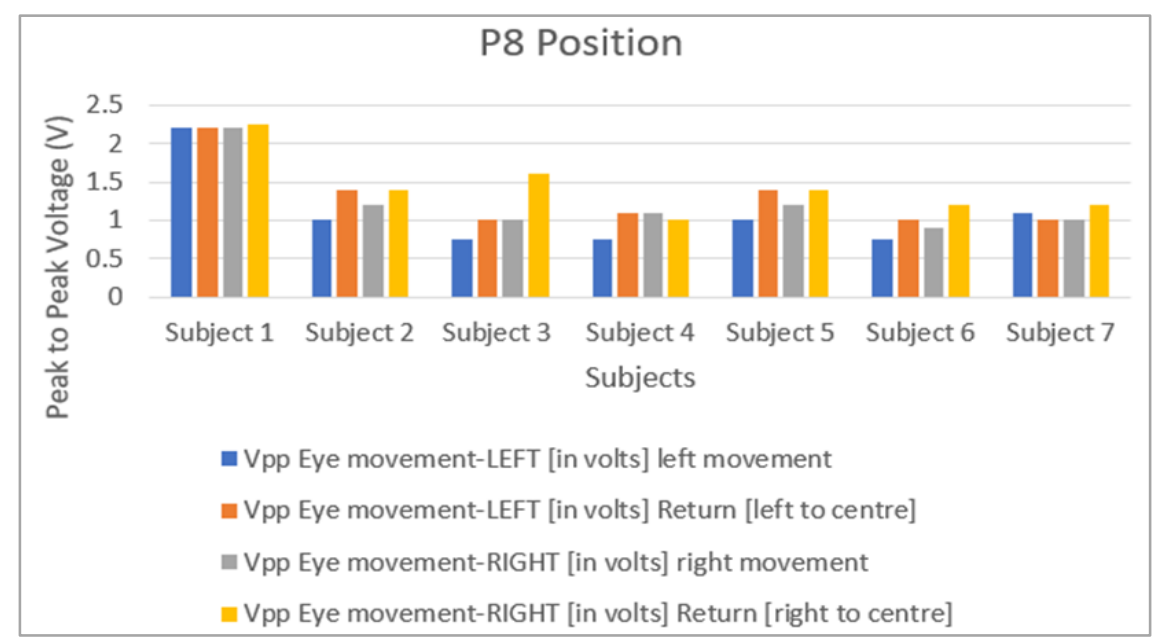

Figure 16 Data for P8 position

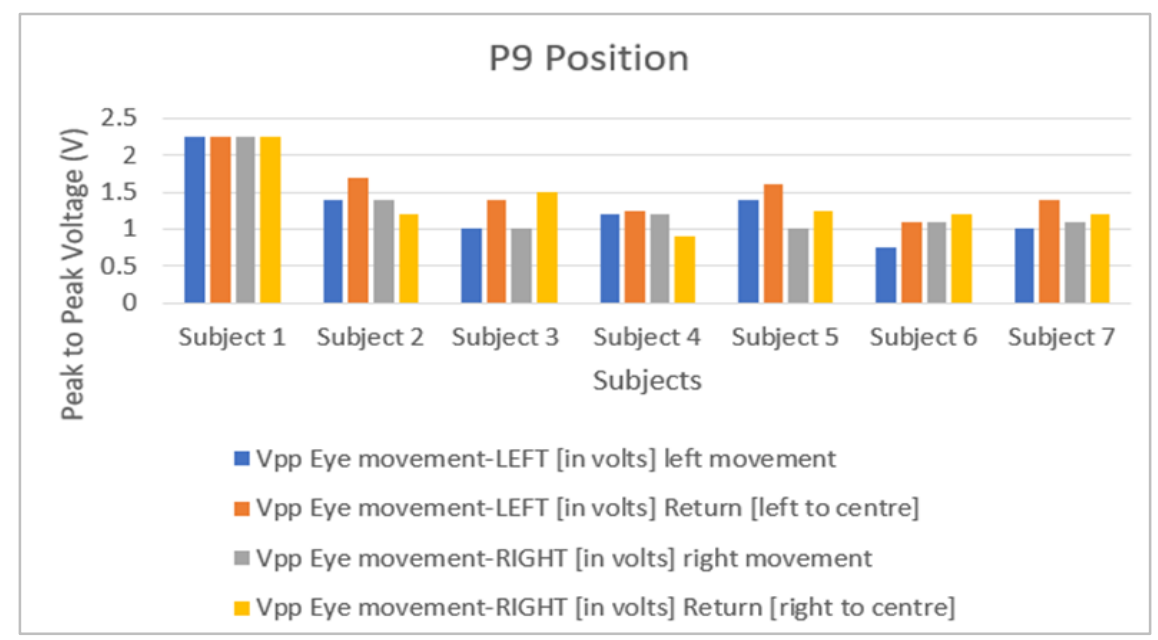

Figure 17 Data for P9 position 
Karthik Raj V

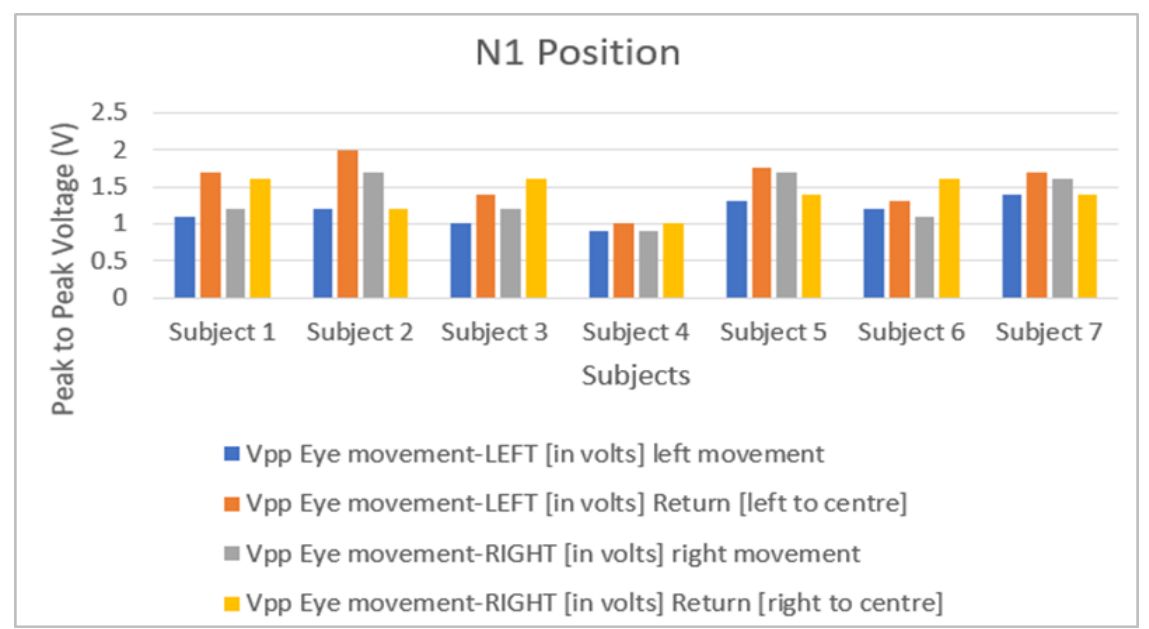

Figure 18 Data for N1 position

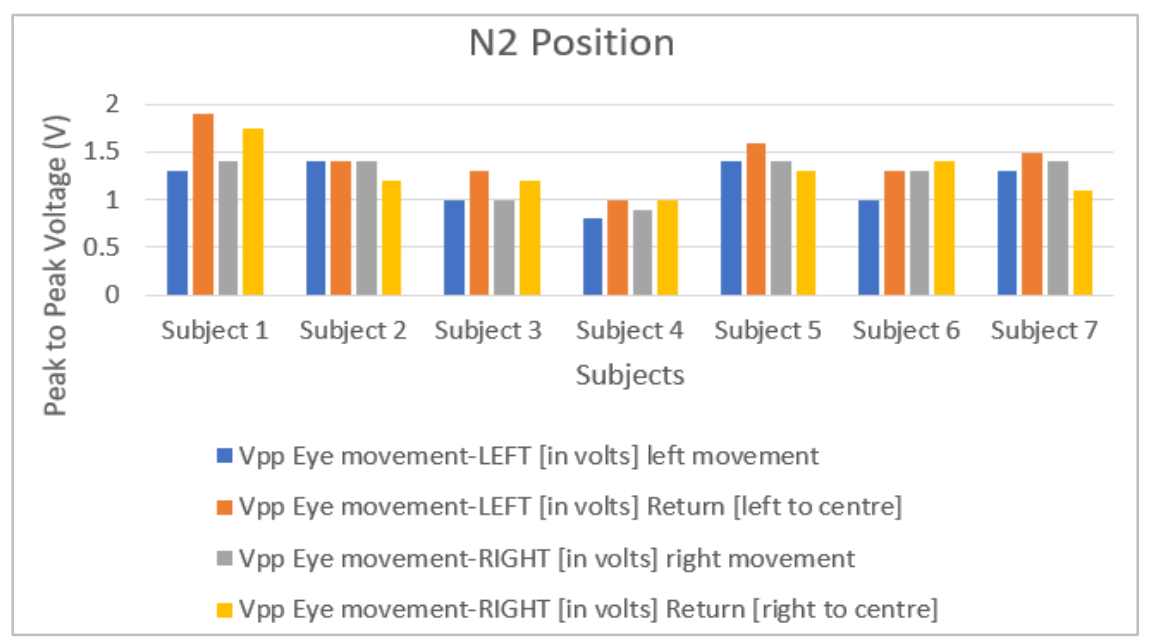

Figure 19 Data for N2 position

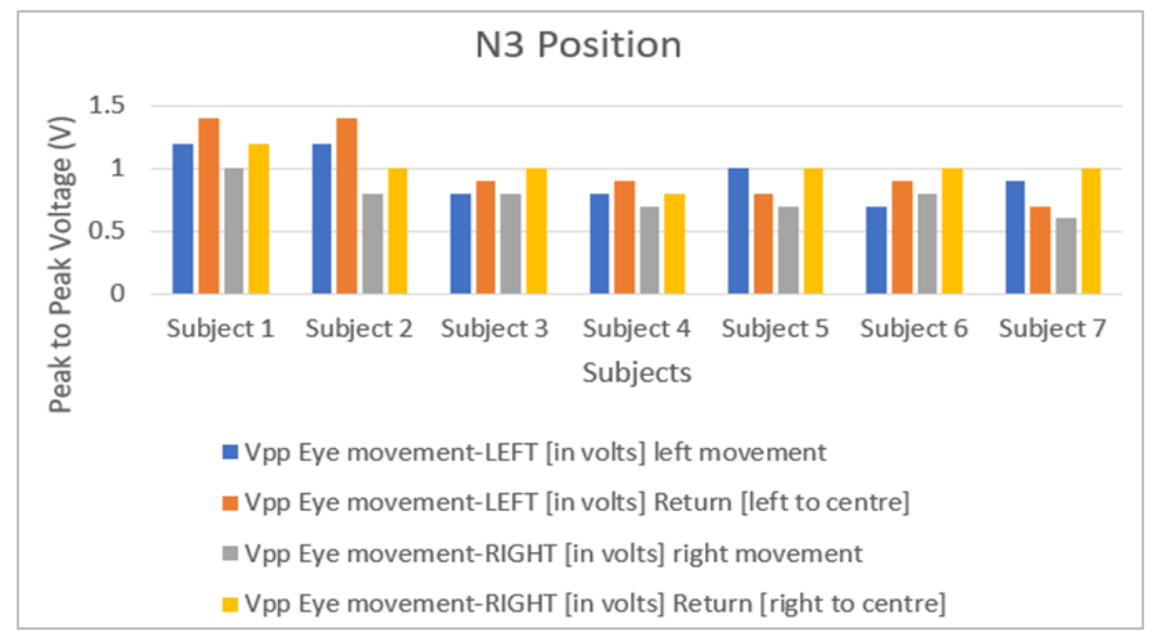

Figure 20 Data for N3 position 


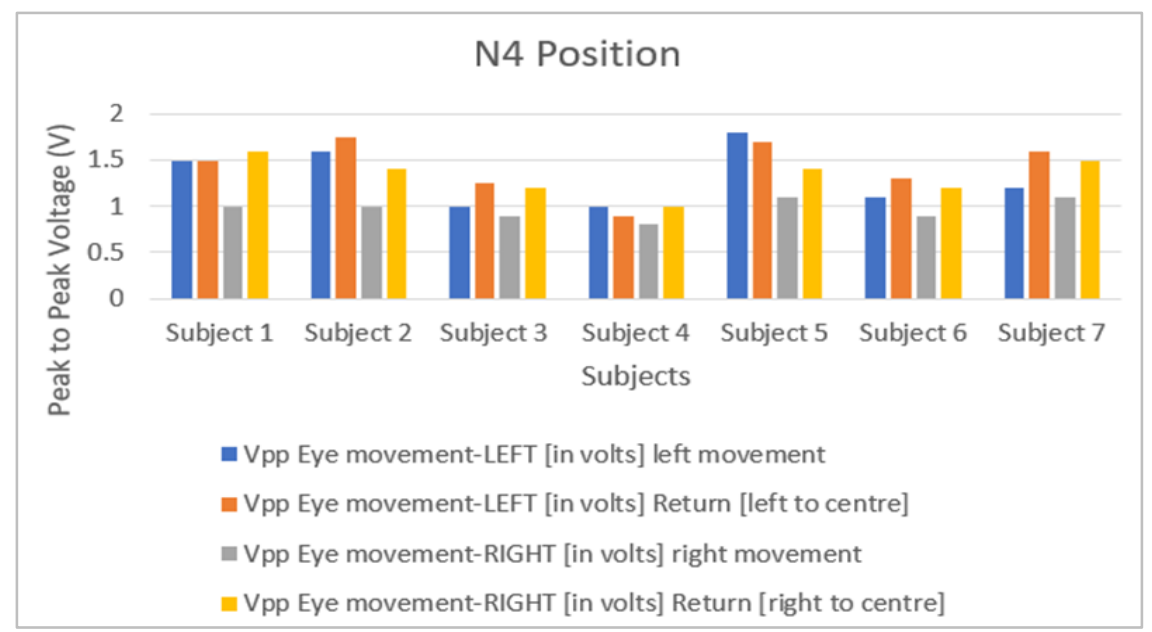

Figure 21 Data for N4 position

\section{Discussion}

The EOG waveforms are picked up effectively. The functioning of the circuit has been verified by its ability to produce clear signals with minimal distortions. The amplitude values in Figures 9, 10, 11, $12,13,14,15,16,17,18,19,20$ and 21 are consistent with that of the EOG signals, thus proving that all applied electrode positions are active sites of signal acquisition that can be used as inputs in biomedical applications. Furthermore, it can be confirmed that the signals are indeed EOGsignals due to the similarity in waveform shape to that of a general EOG signal [6, 14].

The above study is initially performed with nine electrode placements that could give a guaranteed result. This is due to the fact that the basic electrode positions are already confirmed sources of EOG signal. Four new positions, named N1, N2, N3 and N4 are deduced through careful experimentation, keeping in mind that the configurations needed a minimum of facial electrodes to one. If we take a closer look at each of these positions, we can notice that only one electrode is placed on the face at each instance. This electrode is always the positive (+) electrode. On the $\mathrm{N} 1$ and $\mathrm{N} 2$ positions, the positive (+) electrode is placed on the right temple. The negative (-) and reference electrodes are placed away from the face. Similarly, in positions N3 and N4, the positive (+) electrode is placed on the opposite side of the face at the left temple. This switching of sides is what causes an inversion of the peaks, as shown in Figure 8. In the circuit diagram (Figure 2), the positive (+) electrode voltage follower contributes to the positive input of the instrumentation amplifier (pin 3), whereas the negative voltage follower is given to the negative input at pin 2 of the instrumentation amplifier. The positive electrode is primarily set as the electrode in the right side of the face. Thus, movement of the eyeball to the right allows the flow of the positive cycle first and subsequently the negative cycle. This response is homologous to the left movement as the negative electrode gives the negative cycle first in its response due to its placement on the left side of the face. Thus, the effect of electrode placement on signal morphology has been justified.

Observing the amplitude values achieved, as shown in Figures 18, 19, 20 and 21, we could state that the positions N1, N2 and N4 yield the best outputs based on the strength of the signal achieved. The explanation for this result lies in the placement of the reference electrode. Observing the positioning of individual electrodes of N1, N2 and N4, we can see that in N1, the electrode- 2 is placed at the right temple, the electrode-1 is placed at the jawline below the left ear and the ground potential is given on the right earlobe. Similarly, N2 has the same placement for electrode-2, whereas electrode-1 is given behind the left earlobe, while the ground potential is given behind the right earlobe. N4 has a switched position of the electrode- 2 at the left temple while placing electrode- 1 behind the right earlobe and ground potential behind the left earlobe. Through this evidence, we come to the conclusion that in each of these configurations, the reference electrode is situated on the same side of the face as the positive (+) electrode.

Comparing the values achieved by the new positions with those of the original positions, it is observed that the amplitude value is reduced in the new positions. While the difference of the amplitudes itself was not very large, it proved that the positions P1, P2, P3, P4, 
$\mathrm{P} 5, \mathrm{P} 6, \mathrm{P} 7, \mathrm{P} 8$ and $\mathrm{P} 9$ produced the stronger signal and some of these positioning usages were also seen in research papers for various applications [4-6]. This behavior was concurrent with the fact that the placement of positive (+) and negative (-) electrodes at their respective temples produces a stronger signal [4]. Considering the objective of keeping only one electrode on the face, positions N1, N2 and N4 had produced a more than desirable result. However, considering the noisiness of the signals, position N2 did not offer a very reliable signal. In this scenario, only positions $\mathrm{N} 1$ and N4 produced a signal viable enough to be used for assistive device applications. The strength of the signals is high even with only one facial electrode. This was a visible achievement of the positioning used in the configurations. We could also infer that a significant deciding factor of the strength of the signal was the placement of the reference electrode. In three of the four new positions, the ground potential was placed on the same side as the positive (+) electrode; the signal acquired was strong in these three positions. In two of these positions, the noise value was the least. These positions were N1 and $\mathrm{N} 4$ and were the most likely results of the positions considered in the study. Thus, positions N1 and N4 completely fulfilled the objectives of the proposed work by producing a strong EOG signal with only one facial electrode to enhance patient comfort. Also, the total gain required for the $\mathrm{N} 1$ to $\mathrm{N} 4$ was more compared to the total gain required for $\mathrm{P} 1$ to $\mathrm{P} 9$. The average peak to peak amplitude value of the EOG signal for the new site came around about 1.25 volts, whereas the other acquisition sites were more than the 1.25 volts. Complete list of abbreviations is shown in Appendix I.

\subsection{Limitation}

Here we are using wet electrodes, which cannot be used for more extended periods. As a result, it is necessary to change the wet electrodes after some time; otherwise, there will not be proper contact between the skin and the electrodes.

\section{Conclusion and future work}

This research aimed to derive new locations aside from the regular electrode positions to acquire the EOG signal. To discover these, the construction of a low-cost instrumentation circuit to acquire horizontal EOG signals was initiated. The reduction of facial electrodes to one was set as another primary objective to enhance patient comfort and ease of use. Four new positions N1, N2, N3 and N4 were derived, with positions N1, N2 and N4 achieving a stronger output as a direct result of its reference placement on the same side as its positive (+) electrode placement. N1 and N4 produced a low noise signal of these positions and are taken as viable positions for biomedical applications. The system's usage of pre-gelled disposable electrodes offered a stable and almost distortion-free signal output, thus verifying the system's stability. The system can be made wireless for assistive device applications, giving more flexibility and ease of use in the future. Moreover, we can use dry electrodes instead of wet electrodes to be used for more extended periods.

\section{Acknowledgment}

We want to thank the faculty and the Department of Biomedical Engineering, Vel Tech Rangarajan Dr. Sagunthala R \& D Institute of Science and Technology, for their constant support, guidance and resources. We express our gratitude to SRM Institute of Science and Technology and Vel Tech Rangarajan Dr. Sagunthala R \& D Institute of Science and Technology for providing the facilities and infrastructure needed for the performance of the research work.

\section{Conflicts of interest}

The author has no conflicts of interest to declare.

\section{References}

[1] Webster JG. Medical instrumentation: application and design. John Wiley \& Sons; 2009.

[2] Zhang J, Wang B, Zhang C, Hong J. Volitional and real-time control cursor based on eye movement decoding using a linear decoding model. Computational Intelligence and Neuroscience. 2016:111.

[3] Swami P, Gandhi TK. Assistive communication sy stem for speech disabled patients based on electrooculogram character recognition. In international conference on computing for sustainable global development 2014 (pp. 373-6). IEEE.

[4] López A, Ferrero FJ, Valledor M, Campo JC, Postolache O. A study on electrode placement in EOG systems for medical applications. In international symposium on medical measurements and applications 2016 (pp. 1-5). IEEE.

[5] Tarunkumar S, Raghul SS, Karthik RV, Pon V. An assistive device for quadriplegic patients using NIMyRIO. Biomedical Engineering: Applications, Basis and Communications. 2017; 29(3).

[6] Aswin RV, Karthik RV. EOG based low cost device for controlling home appliances. International Journal of Innovative Research in Science, Engineering and Technology . 2014; 3(3):708-11.

[7] Reda R, Tantawi M, Shedeed H, Tolba MF. Eye movements recognition using electrooculography signals. In joint European-US workshop on ap plications of invariance in computer vision 2020 (pp. 490-500). Springer, Cham. 
[8] Barea R, Boquete L, Mazo M, López E. System for assisted mobility using eye movements based on electrooculography. IEEE Transactions on Neural Systems and Rehabilitation Engineering. 2002; 10(4):209-18.

[9] Jambhulkar A, Wandhare S, Baraskar D, Barahate K. Wireless and portable EOG based interface for controlling wheelchair. International Journal of Science, Engineering and Technology . 2016; 5:189-91.

[10] Konwar P, Bordoloi H. A system design approach to control a wheelchair using EOG signal. Current Trends in Technology and Science. 2014; 3(3):155-8.

[11] Usakli AB, Gurkan S. Design of a novel efficient human-computer interface: an electrooculagram based virtual keyboard. IEEE Transactions on Instrumentation and Measurement. 2009; 59(8):2099108.

[12] Gandhi T, Trikha M, Santhosh J, Anand S. Development of an expert multitask gadget controlled by voluntary eye movements. Expert Systems with Applications. 2010; 37(6):4204-11.

[13] Ang AM, Zhang ZG, Hung YS, Mak JN. A userfriendly wearable single-channel EOG-based humancomputer interface for cursor control. In international IEEE/EMBS conference on neural engineering 2015 (pp. 565-8). IEEE.

[14] Mamatha KM, Sumalatha S, Nalini S. EOG based HM I for paralysed people to control electrical devices. International Journal of Engineering Research \& Technology. 2013; 2(5):1319-24.

[15] Bhuyain MF, Shawon MA, Sakib N, Faruk T, Islam MK, Salim KM. Design and development of an EOGbased system to control electric wheelchair for people suffering from quadriplegia or quadriparesis. In international conference on robotics, electrical and signal processing techniques 2019 (pp. 460-5). IEEE.

[16] Kuntal K, Banerjee I, Lakshmi PP. Design of wheelchair based on electrooculography. In international conference on communication and signal processing 2020 (pp. 632-6). IEEE.

[17] Huang Q, He S, Wang Q, Gu Z, Peng N, Li K, et al. An EOG-based human-machine interface for wheelchair control. IEEE Transactions on Biomedical Engineering. 2017; 65(9):2023-32.

[18] Sucres MA, Pérez SS, Zavala IV, Ramírez DL, Hernández NR. EOG-based interface and speech recognition for wheelchair control. In international conference on engineering Veracruz 2019 (pp. 1-4). IEEE.

[19] López A, Fernández D, Ferrero FJ, Valledor M, Postolache O. EOG signal processing module for medical assistive systems. In international sy mposium on medical measurements and applications 2016 (pp. 15). IEEE.

[20] Aziz S, Ibraheem S, Malik A, Aamir F, Khan MU, Shehzad U. Electrooculugram based communication system for people with locked-in-syndrome. In international conference on electrical, communication, and computer engineering 2020 (pp. 1-6). IEEE.
[21] Kołodziej M, Tarnowski P, Sawicki DJ, Majkowski A, Rak RJ, Bala A, et al. Fatigue detection caused by office work with the use of EOG signal. IEEE Sensors Journal. 2020; 20(24):15213-23.

[22] Milanizadeh S, Safaie J. EOG-based HCI system for quadcopter navigation. IEEE Transactions on Instrumentation and Measurement. 2020; 69(11):89929.

[23] De LSE, Conzelmann P. Computer USB-mouse emulation using EOG. In international sy mposium on instrumentation systems, circuits and transducers 2019 (pp. 1-5). IEEE.

[24] Hay awi AA, Waleed J. Driver's drowsiness monitoring and alarming auto-system based on EOG signals. In international conference on engineering technology and its applications 2019 (pp. 214-8). IEEE.

[25] Hou HK, Smitha KG. Low-cost wireless electrooculography speller. In international conference on systems, man, and cybernetics 2018 (pp. 123-8). IEEE.

[26] Latifoğlu F, İleri R, Demirci E, Altıntop ÇG. Detection of reading movement from EOG signals. In international symposium on medical measurements and applications 2020 (pp. 1-5). IEEE.

[27] Lin CT, King JT, Bharadwaj P, Chen CH, Gupta A, Ding W, et al. EOG-based eye movement classification and application on HCI baseball game. IEEE Access. 2019; 7:96166-76.

[28] Lu YY, Huang YT. A method of personal computer operation using electrooculography signal. In Eurasia conference on biomedical engineering, healthcare and sustainability 2019 (pp. 76-8). IEEE.

[29] Banerjee A, Chakraborty S, Das P, Datta S, Konar A, Tibarewala DN, et al. Single channel electrooculogram (EOG) based interface for mobility aid. In international conference on intelligent human computer interaction 2012 (pp. 1-6). IEEE.

[30] Mala S, Latha K. Feature selection in classification of eye movements using electrooculography for activity recognition. Computational and $\mathrm{M}$ athematical Methods in Medicine. 2014:1-9.

[31] Vidal M, Bulling A, Gellersen H. Analysing EOG signal features for the discrimination of eye movements with wearable devices. In proceedings of the international workshop on pervasive eye tracking \& mobile eye-based interaction 2011 (pp. 15-20).

[32] Bulling A, Ward JA, Gellersen H, Tröster G. Eye movement analysis for activity recognition using electrooculography. IEEE Transactions on Pattern Analysis and Machine Intelligence. 2010; 33(4):74153.

[33] Mala S, Latha K. Classification of electrooculograph signals: comparing conventional classifiers using CBFS feature selection algorithm. In fourth international conference on computing, communications and networking technologies 2013 (pp. 1-7). IEEE.

[34] Yamagishi K, Hori J, Miyakawa M. Development of EOG-based communication sy stem controlled by eightdirectional eye movements. In international conference 
of engineering in medicine and biology society 2006 (pp. 2574-7). IEEE.

[35] Nann M, Cordella F, Trigili E, Lauretti C, Bravi M, Miccinilli S, et al. Restoring activities of daily living using an EEG/EOG-controlled semiautonomous and mobile whole-arm exoskeleton in chronic stroke. IEEE Sy stems Journal. 2020; 15(2):2314-21.

[36] Golparvar AJ, Yapici MK. Graphene-coated wearable textiles for EOG-based human-computer interaction. In international conference on wearable and implantable body sensor networks 2018 (pp. 189-92). IEEE.

[37] Xiao J, Qu J, Li Y. An electrooculogram-based interaction method and its music-on-demand application in a virtual reality environment. IEEE Access. 2019; 7:22059-70.

[38] Zhang R, He S, Yang X, Wang X, Li K, Huang Q, et al. An EOG-based human-machine interface to control a smart home environment for patients with severe spinal cord injuries. IEEE Transactions on Biomedical Engineering. 2018; 66(1):89-100.

[39] Zheng WL, Gao K, Li G, Liu W, Liu C, Liu JQ, et al. Vigilance estimation using a wearable EOG device in real driving environment. IEEE Transactions on Intelligent Transportation Systems. 2019; 21(1):17084.

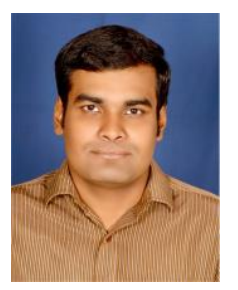

Karthik Raj V had received an M.E degree in Medical Electronics from College of Engineering, Anna University in 2011 and a B.Tech in Biomedical Engineering from Sahrdaya College of Engineering and Technology, Calicut University in 2008. His B.E project of Voice-Controlled Wheel Chair was recognized as the Best Project of the Department of Biomedical Engineering in Sahrday a College of Engineering and Technology in 2008. He is currently working as an Assistant Professor in the Department of Biomedical Engineering, Vel Tech Rangarajan Dr. Sagunthala R \& D Institute of Science and Technology, India. His research interests include Bio-instrumentation and Medical Electronics

Email: karthikhrajv@gmail.com

\begin{tabular}{lll}
\multicolumn{2}{l}{ Appendix I } \\
\hline S. No. & Abbre viation & Description \\
\hline 1 & CMRR & Common-Mode Rejection Ratio \\
\hline 2 & DF & Damping Factor \\
\hline 3 & DSO & Digital Storage Oscilloscope \\
\hline 4 & EOG & Electrooculography \\
\hline 5 & $\mathrm{~Hz}$ & Hertz \\
\hline 6 & $\mathrm{k} \Omega$ & kiloohm \\
\hline 7 & LabVIEW & $\begin{array}{l}\text { Laboratory Virtual Instrument } \\
\text { Engineering Workbench }\end{array}$ \\
\hline 8 & $\mathrm{mV}$ & milli-volt \\
\hline 9 & $\mu \mathrm{F}$ & microfarad \\
\hline 10 & $\mathrm{NI}-$ ELVIS & $\begin{array}{l}\text { National Instruments-Educational } \\
\text { Laboratory Virtual Instrumentation } \\
\end{array}$ \\
& & Suite \\
\hline 11 & NI-DAQ & National Instruments-Data Acquisition \\
\hline 12 & Op-amp & Operational amplifier \\
\hline
\end{tabular}

